\title{
Biomedical Applications of Nanodiamonds: An Overview
}

\author{
D. Passeri ${ }^{1}$, F. Rinaldi ${ }^{2}$, C. Ingallina ${ }^{3}$, M. Carafa ${ }^{2}$, M. Rossi ${ }^{1,4}$, \\ M. L. Terranova ${ }^{5}$, and C. Marianecci ${ }^{2}, *$ \\ ${ }^{1}$ Department of Basic and Applied Sciences for Engineering, Sapienza, \\ University of Rome, via A. Scarpa 16, 00161 Roma, Italy \\ ${ }^{2}$ Department of Drug Chemistry and Technologies, Sapienza University of Rome, \\ Piazzale A. Moro 5, 00185, Rome, Italy \\ ${ }^{3}$ Center for Life Nano Science@Sapienza, Istituto Italiano di Tecnologia (IIT), \\ Viale Regina Elena 291, 00185, Rome, Italy \\ ${ }^{4}$ Center of Nanotechnologies for Engineering (CNIS), Sapienza University of Rome, \\ Piazzale A. Moro 5, 00185, Rome, Italy \\ ${ }^{5}$ Department of Chemical Sciences and Technologies, University of Rome 'Tor Vergata,' \\ via della ricerca Scientifica, 00133, Rome, Italy
}

\begin{abstract}
Nanodiamonds are a novel class of nanomaterials which have raised much attention for application in biomedical field, as they combine the possibility of being produced on large scale using relatively inexpensive synthetic processes, of being fluorescent as a consequence of the presence of nitrogen vacancies, of having their surfaces functionalized, and of having good biocompatibility. Among other applications, we mainly focus on drug delivery, including cell interaction, targeting, cancer therapy, gene and protein delivery. In addition, nanodiamonds for bone and dental implants and for antibacterial use is discussed. Techniques for detection and imaging of nanodiamonds in biological tissues are also reviewed, including electron microscopy, fluorescence microscopy, Raman mapping, atomic force microscopy, thermal imaging, magnetic resonance imaging, and positron emission tomography, either in vitro, in vivo, or ex vivo. Toxicological aspects related to the use of nanodiamonds are also discussed. Finally, patents, preclinical and clinical trials based on the use of nanodiamonds for biomedical applications are reviewed.
\end{abstract}

Keywords: Nanodiamond, Biomedicine, Drug Delivery, Diagnostic, Imaging, Therapy, Theranostic.

\section{CONTENTS}

1. Introduction

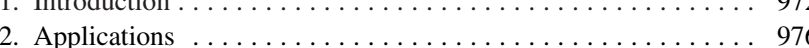

2.1. Cell Interaction Studies and Cell Receptor Targeting . . . . 976

2.2. $\mathrm{pH}$ Sensitive Targeting . . . . . . . . . . . . . . . . . . . . 977

2.3. Cancer Therapy ... . . . . . . . . . . . . . . . . . . . 978

2.4. Antibacterial Use . . . . . . . . . . . . . . . . . . . . . . 980

2.5. Immunogenic Effect . . . . . . . . . . . . . . . . . . . . 981

2.6. Gene Therapy . . . . . . . . . . . . . . . . . . . . . 981

2.7. Protein Delivery . . . . . . . . . . . . . . . . . . . . . 982

2.8. Bone Implants . . . . . . . . . . . . . . . . . . . . . . 983

2.9. Imaging . . . . . . . . . . . . . . . . . . . . . . 983

2.10. Toxicological Aspects . . . . . . . . . . . . . . . . 984

3. NDs Based Patents, Preclinical and Clinical Trials . . . . . . . . 985

4. Concluding Remarks and Perspectives . . . . . . . . . . . . . . 986

References and Notes . . . . . . . . . . . . . . . . . 986

*Author to whom correspondence should be addressed.

\section{INTRODUCTION}

In the last fifty years, the studies on preparation, characterization and applications of non conventional new drug or diagnostic molecules delivery systems (DS) are gaining ever increasing importance. The use of DS allows to face the major issues in drug or diagnostic molecule release: (1) unfavorable pharmacokinetics and biodistribution which lead to unwanted side effects (e.g., chemotherapy), (2) early drug degradation in the bloodstream by reticulo-endothelial system (RES), and (3) inefficient uptake at target sites that leads to low drug or low imaging agent efficacy in cells. In this sense, nanocarriers that incorporate drug or imaging agents or both, offer an innovative approach in the field of delivery, providing a range of features including cargo protection and increased dose delivery to target sites. Actually, the 
complexity of human diseases may lead to ineffective in vivo drug treatments, consequently the development of biologically functional nanocarriers that incorporate drugs or imaging agents to selectively bind and target specific cells becomes crucial. There have been many attempts to functionalize and design nanocarriers so as to increase their efficacy; nevertheless, although the criteria for a successful nanocarrier are quite numerous, only few nanocarriers have actually shown significant clinical potential. By molding nanomaterials numerous nanocarriers (Fig. 1) have been developed to securely deliver drugs and various other therapeutic and diagnostic agents specifically into target sites. Many of the conventional nano DS (e.g., liposomes, micelles, and polymer-based nanodevices) have reached the late stages of development, and some of them were approved. Over the last two decades,
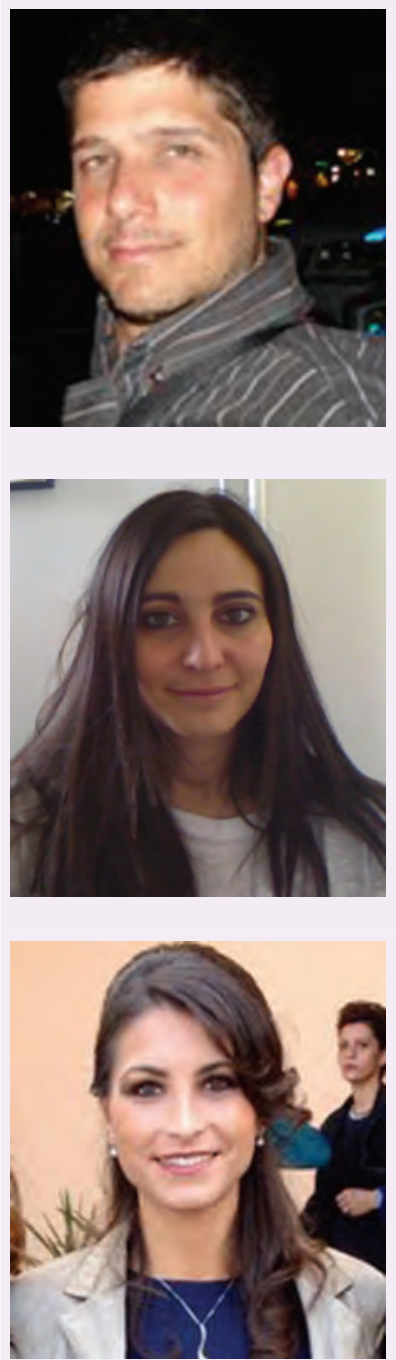

drugs. Author of 2 resear

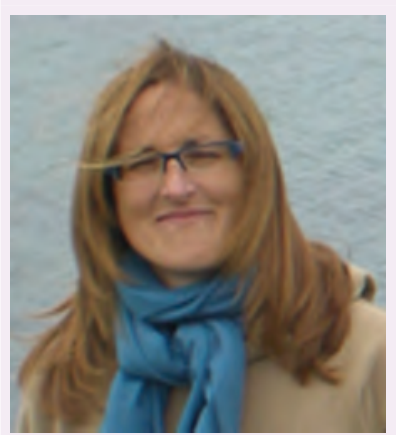

D. Passeri, postdoctoral research fellow at Sapienza University of Rome (Italy), Faculty of Civil and Industrial Engineering. MSc in Electronic Engineering and Ph.D. in Electromagnetism. His research activity has been mainly focused on scanning probe microscopy techniques for the characterization of physical parameters of nanomaterials at the nanoscale. In particular, he is expert in mechanical characterizations using atomic force microscopy based acoustic techniques. Author of about 40 publications in international peer-reviewed journals and books. Editor of a book on acoustic scanning probe microscopy. Presenting or contributing author of about 50 oral communications or posters at international conferences. Reviewer for about 20 journals focused on materials science, nanotechnology, and microscopy. He is member of the European Microscopy Society and of the Società Italiana di Scienze Microscopiche.

F. Rinaldi, research grant in Pharmaceutical Technology at Faculty of Pharmacy and Medicine, Department of Drug Chemistry and Technologies, "Sapienza" University of Rome. Her research activity is mainly focused on the study of phospholipid and non phospholipid vesicles as drug delivery systems, in particular: preparation and characterization of vesicles for cytoplasmic delivery of therapeutic agents ( $\mathrm{pH}$-sensitive niosomes) and for topic (various niosomal formulations), oral (coated liposomes), pulmonary (coated and uncoated niosomes) and brain (liposomes-niosomes) delivery of drugs. Expert in physical-chemical characterization by fluorimetric techniques of different vesicular system. Author of about 15 publications in international peer-reviewed journals and books. Presenting or contributing author of about 20 oral communications or posters at international conferences.

C. Ingallina, Master Degree in Medicinal Chemistry at Facoltà di Farmacia, "Sapienza" Università di Roma in 2010 in Organic Chemistry. In 2011 applied Leonardo Da Vinci Programme, worked for six months in Medichem, Spain, on process chemistry development and optimization for APIs production. From 2012 until now Ph.D. student in Medicinal Chemistry at Facoltà di Farmacia, Sapienza, Università di Roma. Her research activity is mainly focused on Organic Chemistry: isolation, purification and characterization of natural products by HPLC-UV, LC-MS, NMR; synthesis of natural product analogs with antitumor activity, particularly involved in the Hedgehog pathway as inhibitor of brain tumors; and on Pharmaceutical Technology: preparation and characterization of non-phospholipid (niosomes) vesicular systems; studies on cell internalization of niosomal nanocarriers through the blood brain barrier; application of niosomes for brain delivery of papers, one Italian patent, one book chapter and 9 congress presentations.

M. Carafa, Since 2008 Associate Professor of Pharmaceutical Technology - Faculty of Pharmacy and Medicine, University "Sapienza," Rome. Personal skills and competences: preparation and characterization of vesicular systems: phospholipid and surfactant vesicles and $\mathrm{pH}$-sensitive vesicles as drug delivery systems for several pharmaceutical applications: topical, ophthalmic, diagnostic, pulmonary, oral, drug delivery in CNS disorders and cellular targeting. Deep physico-chemical characterization of vesicular formulations as drug delivery systems and diagnostic agents. The scientific activity of Prof. Carafa is attested by 55 papers, 3 patents and more than 200 congress presentations. 


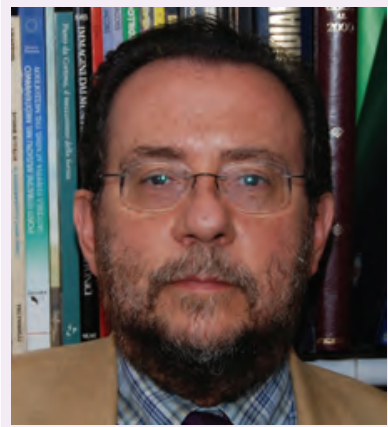

M. Rossi, Professor of Physics at Sapienza University of Rome (Italy), coordinates the scientific activity of the Electron Microscopies and Nanoscopies Laboratory (EMINA) at the Department of Basic and Applied Sciences for Engineering. He is member of the Scientific Board of Centre of Research for Nanotechnologies applied to Engineering of Sapienza University of Rome (CNIS). He teaches 'General Physics' and 'Nanocharacterization Techniques and Microscopies.' He is member of the Doctorate Board in Mathematical Model, Nanosciences and Electromagnetism. His present research activity is primarily aimed at the study of C-based nanomaterials and their possible applications, using scanning probe and electron microscopies, X-Ray and electron diffraction techniques as means to investigate structural, functional and morphological features of the nanomaterials of interest. He is co-author of 158 papers in peer-reviewed journals and of 1 patent; co-editor of 1 book and 10 volumes of proceedings.

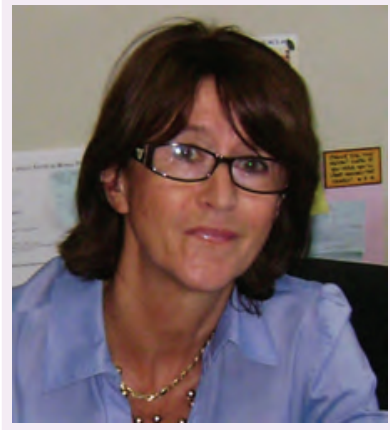

M. L. Terranova, Professor of Chemistry at the University Tor Vergata (Roma, Italy), heads the Minima lab at the Department of Chemical Science and Technology. She teaches 'Chemistry,' 'Solid State Characterizations' and 'Nanostructured Materials.' She has extensive expertise on different scientific topics, including nuclear chemistry, nuclear physics, laserinduced reactions in gas-phase, ion- and laser-induced modifications in solids, electrochemical and vapour deposition processes. Her present research activity is mainly focused to the synthesis, processing and functional testing of nanomaterials: carbon nanostructures (nanodiamonds, nanotubes, graphenes, onions), nanocomposites and hybrid organic/inorganic structures. The materials and the assembled systems are produced for applications in micro/nano-electronics, optoelectronics, energetics, sensing, thermal management and biorelated nanotechnologies. MLT co-authored 280 papers, 4 books and 4 patents.

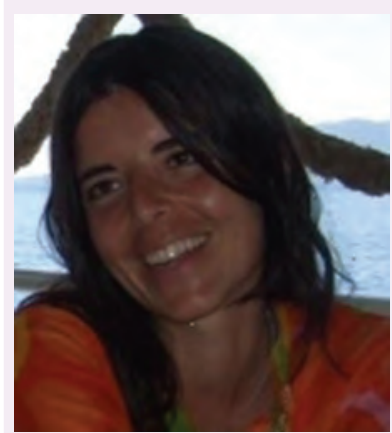

C. Marianecci, Assistant professor at Faculty of Pharmacy and Medicine, Department of Drug Chemistry and Technologies, "Sapienza" University of Rome. Her research activity is mainly focused on of Pharmaceutical Technology and in particular on: preparation and characterization of phospholipid (liposomes) and non-phospholipid (niosomes) not $\mathrm{pH}$ and $\mathrm{pH}$-sensitive vesicular systems; studies on cell internalization pathway of liposomal and niosomal nanocarriers in fibroblasts, macrophagic cells and granulocytes, monocytes and linfocytes; application of niosomes for topical, pulmonary and brain delivery of drugs; study on L-Dopa delivery and DNA delivery by different liposomal formulations; deep chemical-physical characterization studies on vesicular nanocarriers and preparation and characterization of theranostic nanocarriers. Author of about 50 national and international research papers, 2 patents, 3 book chapters and about 60 oral and poster presentations. Referee for 10 international journals focused on nanotechnology and drug delivery, member of the Editorial Board of the "World Journal of Respirology."

the development of synthesis and characterization techniques including the ability to manipulate molecules and supramolecular structures for beneficial functions blossomed into innovative-engineered nanomaterials suitable for biopharmaceutical/therapeutic/diagnostic applications. Another important development is that nanocarriers are becoming highly integrated and multifunctional so as to include a range of applications such as on demand release, specific tissue/cell type targeting, in vivo imaging and diagnosis, and photothermal treatment. ${ }^{1}$ The ultimate goal of nano DS is to generate therapeutically, diagnostically and clinically useful formulations for disease treatments or diagnoses in patients. While actively studied in academia and industry, the pursuit of optimized nanocarriers is in continuous evolution and the topic requires innovation and skill in order to translate them into human trials.
A successful nanocarrier should ideally meet the following requirements:

(1) formulation with biocompatible/biodegradable/bioexcretable materials,

(2) high drug and cargo loading capacity,

(3) site-specific delivery mechanism to avoid normal cells and tissues,

(4) zero or negligible premature drug release, and

(5) controlled release mechanism to provide an effective dose to the target site.

To date, there is a very limited number of nanocarriers which can achieve such prerequisites ${ }^{2}$ and in particular most of the potential products have been based on liposomes and polymeric materials while ignoring the potentialities of inorganic materials with unique physiochemical properties. In particular, carbon allotropes such 


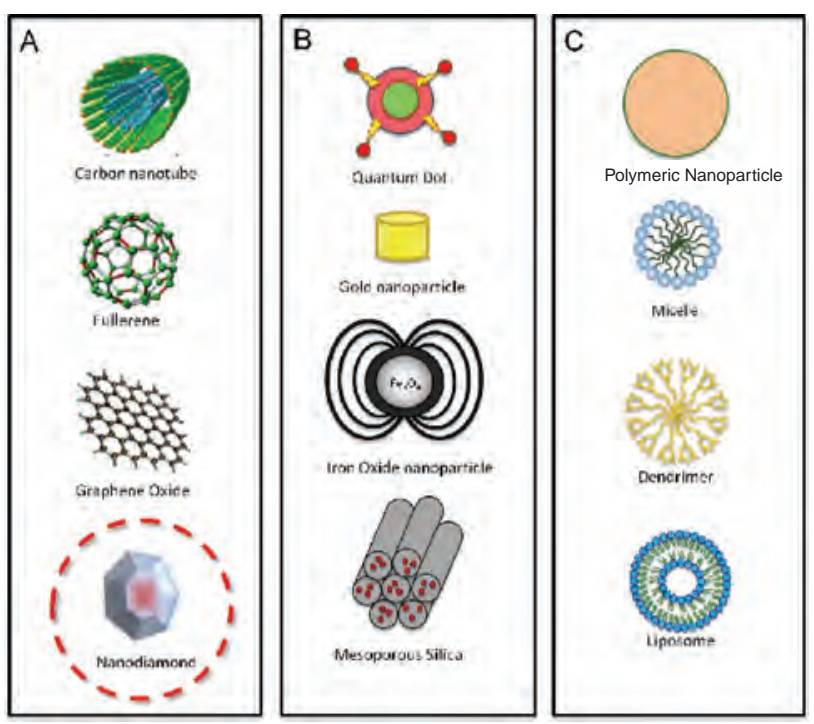

Figure 1. Sketch of the leading nanocarriers for drug delivery: (A) carbon based inorganic nanocarriers; (B) other inorganic nanocarriers; (C) organic nanocarriers.

as graphene oxide, carbon nanotubes, and nanodiamonds (NDs) have similar unique features suitable for being used as diagnostic and therapeutic delivery nanocarriers. The carbon-based materials have sufficient surface-to-volume ratio, thermal conductivity, rigid structural properties capable of post-chemical modification, and excellent biocompatibility. These NDs are very versatile and are able to deliver water insoluble drugs, antigens, antibodies, nucleic acids and imaging agents into target cells, where the therapeutic or imaging/diagnostic molecules would be released, and efficiently utilized. These systems can be also easily scaled up for an industrial production and easily modificated to create a DS equipped with a set of designed functionalities. NDs simply seem similar to very small diamonds, but they are synthesized by an inexpensive large scale synthesis based on the detonation of carboncontaining explosives. The first records of nanosized diamond particle production come from the Union of Soviet Socialist Republics in the 1960s. However, until the mid1980s they remained unrecognized, when they found use in wear-resistant coatings and as an anti-wear additive for motor oil. The first report of NDs production in the United States did not arrive until 1988, but today, numerous methods for commercially producing diamond nanoparticles ranging from the purification of detonation soot (reviewed in Refs. [3-5]) and laser ablation to milling down larger diamond micro-particles are available. However, since 1990s researchers began to take interest in NDs for biomedical applications. Since then, the field has expanded significantly, with NDs finding use in a multitude of applications from nanoscale magnetic resonance imaging to cancer therapy. ${ }^{6-13}$ The constantly growing interest in NDs and their applications in biomedicine is reflected in the increase of the number of publications in

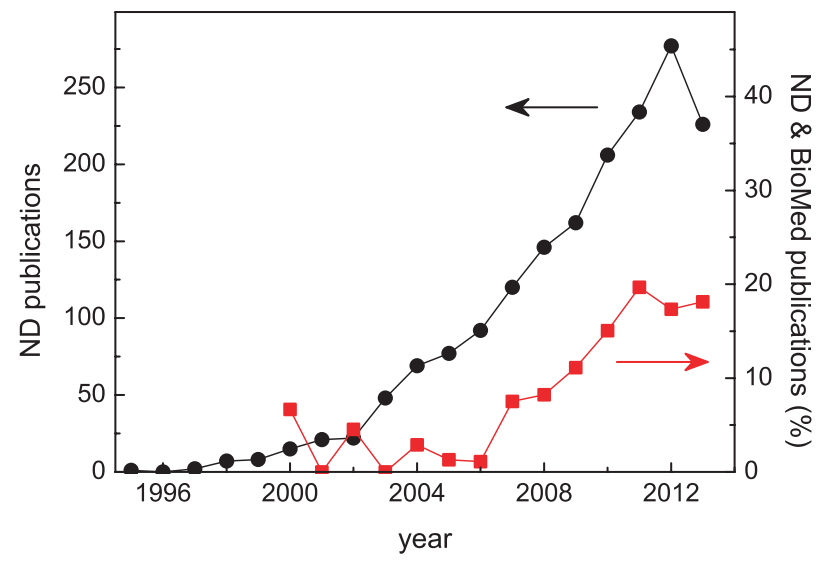

Figure 2. Number of articles published in each year retrieved through a scientific search engine (Scopus) searching the word 'nanodiamond' in the title, abstract, or keywords (black circles, which refer to 'ND publications'). The number of articles addressing biomedical applications of NDs (red squares, which refer to 'ND and BioMed publications') is obtained by adding 'drug delivery,' 'diagnostic,' 'imaging' or 'theranostic.'

such a field. As an example, Figure 2 shows the number of articles published in each year retrieved through a scientific search engine (Scopus) searching the word "nanodiamond' in the title, abstract, or keywords (black circles). One can observe that the number of published articles has increased ten fold in the last ten years. Moreover, the number of articles addressing biomedical applications of NDs can be obtained by adding 'drug delivery,' 'diagnostic,' 'imaging' or 'theranostic' among the words to search for. No articles are obtained before 2000. Nevertheless, since 2000 the ratio between the number of such articles and the total number of articles obtained searching only 'nanodiamonds' (red squares) has constantly increased, reaching about the $20 \%$ in recent years.

Based upon their primary particle sizes, Shenderova and McGuire classified NDs into nanocrystalline particles (1 to $150 \mathrm{~nm}$ ), ultrananocrystalline particles ( 2 to $10 \mathrm{~nm}$ ), and diamondoids (1 to $2 \mathrm{~nm})$. Ultrananocrystalline diamond particles are of particular interest for biomedical applications and detonation NDs with a primary particle size of 4 to $5 \mathrm{~nm}$ are the most interesting. ${ }^{5,14}$ NDs can be classified on the basis of synthetic production methods such as: chemical vapor deposition diamonds, high-pressure high-temperature diamonds (HPHT), and detonation NDs (DNDs). In the context of biological and medical applications, diamond-based platforms including NDs produced via detonation and HPHT, ultra-/nanocrystalline diamond films (UNCD/NCD), and single/polycrystalline diamond films. Although the size, shape and surface properties of NDs are determined by the nature of preparation technique and purification conditions, their structure can be summarized in a core and shell model. The inert core is composed by the diamond carbon while the surface shell is partially comprised of graphitic structures. A wide variety of functional groups such as carboxyl, hydroxyl, lactone, 
anhydride, ketone, and ether can be added on the surface of NDs. These structures can be characterized by $\mathrm{X}$-ray diffraction, able to determine their size, structure, and composition.

Some articles and books can be found in literature where synthetic and functionalization processes as well as applications of NDs are reviewed in details, which we consider and recommend to the reader as landmarks in the field. ${ }^{4,5,13-20}$ The aim of this review is to give a synthetic general overview and depict the state of the art as much up-to-date as possible in the use of NDs for biomedical applications. In particular as biomedical application is meant their use in drug delivery and imaging for in vitro and in vivo studies. The toxicological aspect that affect their safe employment will be also discussed. Finally, the future directions and challenges of NDs research in engineering, medicine, and biotechnology will be considered.

\section{APPLICATIONS}

The application of NDs in biomedical field requires the ability to obtain stable dispersions in an opportune formulation medium, to conjugate with biomolecules, to show inherent biocompatibility, and to reach target tissues and cells where deliver the therapeutic or imaging agent. The wide surface area of biocompatible NDs, with several surface functionalities, is ideal for conjugating various biochemical entities. The suitability of the carrier is highly dependent from its loading capacity as well as from its ability to protect and maintain the therapeutic and imaging properties of the attached entities. A high loading capacity means a high concentration of payload to be delivered using lower concentration of DS itself. At the same time, to the loading capacity, the release of payload from the carrier is also important. The feasibility of being able to tune the release of loaded material from the carrier is advantageous in developing novel applications of these delivery agents, such as controlledand sustainedrelease delivery. NDs are being investigated globally for improving the intracellular delivery of small molecules and biotechnological products, with a major focus on chemotherapeutic agents. In addition to drug delivery, NDs are being widely investigated for imaging applications. Both detonation NDs and HPHT NDs have offered important advantages in this area because HPHT NDs, which can be implanted with nitrogen vacancy centers, have been shown to be remarkably photostable while maintaining biocompatibility. ${ }^{19}$ Although the bonding of drugs or imaging agents have been made by chemical reactions, it is also possible to obtain physical adsorption procedures. The physical interactions avoid the use of complex chemical reactions, which, are of high cost, and can also cause structural alterations of the attached molecule. ${ }^{20}$ The complexity of the NDs aggregates as delivery systems is continuing to evolve with the goal of simplify these systems and achieve controlled release of immobilized substances by specialized enzymes such as lipases, esterases, and proteases. Fundamental is the retention of the NDs-biomolecule complex conformation and functionality to maintain the delivery and targeting characteristics. NDs can be synthesized at low cost (i.e., detonation synthesis) and in large quantities, while their particle and film forms have exhibited high biocompatibility and low toxicity and they are not attacked by body's immune system. Although NDs have recently become commercially available (Alit, Diamond Center, Sinta, Real Dzerzinsk, ITC, Nanoblox, Aldrich, and others), there remains a large variety of size distributions and purities with variable uniformity. ${ }^{16}$ In addition, the unique optical properties and the relative easiness of functionalization, together with good biocompatibility, make NDs particularly suitable as labels for different imaging techniques (e.g., confocal fluorescence microscopy, Raman mapping, electron microscopy). ${ }^{16,18,21,22}$

\subsection{Cell Interaction Studies and Cell Receptor Targeting}

Biologically active molecules exploit many mechanisms and for most of them the passage through the cell membrane in a cell-type or tissue-specific manner is needed, especially when the pharmacological target is located intracellularly. In this case, a complex series of interactions with the cells are required. The way that the therapeutic molecule must generally go through is:

(1) cross one or various biological membranes (e.g., mucosa, epithelium, endothelium) before

(2) diffusing through the plasma membrane to

(3) finally gain access to the appropriate organelle where the biological target is located. ${ }^{23}$

From what above mentioned it is clear that an analysis of NDs uptake by cells is fundamental, but less explored particularly at the cellular and sub-cellular levels.

A number of studies have demonstrated the ability of NDs to internalize into cells. ${ }^{20,24-28}$ The cell internalization of NDs could be dependent upon their physicochemical characteristics such as: surface characteristics, size, shape, and aggregation degree or other parameters like incubation time and concentration. Faklaris et al. demonstrated that NDs having an average size of $46 \mathrm{~nm}$ internalize into HeLa cells predominantly by clathrin-mediated endocytosis after 2 hours of incubation, ${ }^{26}$ on the contrary NDs of $100 \mathrm{~nm}$ internalize into cancer and stem cells after 4 hours of incubation via macropinocytosis. ${ }^{29}$ These results are in agreement to what reported in literature about the dependence of cellular entry mechanism to nanocarrier dimension. ${ }^{30}$ The intracellular localization of the NDs suggests that they have the potential to be employed for intracellular delivery of therapeutic molecules (Table I). Only in few cases the nuclear localization of NDs has been reported, the most showed only perinuclear localization. The nuclear delivery is fundamental when DNA 
Table I. NDs in cell interaction studies and targeting.

\begin{tabular}{llcc}
\hline Composition & \multicolumn{1}{c}{ Cell studies } & Results \\
\hline NDs (size 100 nm) & $\begin{array}{c}\text { Cancer cells A549 and non cancer cells } \\
\text { HFL1 and Beas-2b }\end{array}$ & Clathrin dependent endocytosis \\
$\begin{array}{l}\text { NDs lipid hybrids with EGFR } \\
\text { Cancer cells }\end{array}$ & Enhance internalization \\
$\begin{array}{l}\text { Fluorescein labeled dT-nucleotide for PTX } \\
\text { delivery together with an anti-EGFR } \\
\text { monoclonal antibody on NDs surface } \\
\text { sulfo-LC-SPDP }\end{array}$ & $\begin{array}{c}\text { EGFR-overexpressing MDA-MB-231 } \\
\text { cancerous cells }\end{array}$ & Selective internalization \\
$\begin{array}{l}\text { ND-IgGI125 and RAM-ND BSAI125 } \\
\text { complexes }\end{array}$ & $\begin{array}{c}\text { Sepharose 6B matrix with antigen } \\
\text { immoblized }\end{array}$ & $\begin{array}{c}\text { Derivatized NDs bind to the target antigen } \\
\text { immobilized on Sepharose 6B matrix } \\
\text { through antibody-antigen interaction }\end{array}$ \\
\hline
\end{tabular}

is the loaded material. ${ }^{20}$ In an interesting study Perevedentseva et al. ${ }^{31}$ compare NDs internalization in human cancer and non-cancer cells. In particular, they study the peculiarities and describe quantitatively the difference of NDs interaction with these two kinds of cells. The uptake of NDs (with particles size of $100 \mathrm{~nm}$ ) by different cell lines was analyzed; human lung carcinoma cell (A549) and two kinds of non cancer cells: human lung fibroblast cell (HFL1) and human bronchial epithelial cell (Beas-2b). The NDs inside cells were visualized via confocal fluorescence imaging. The mechanisms of NDs uptake were analyzed via alternatively blocking different possible endocytosis pathways. NDs penetrate into both cancer and non-cancer cells predominantly via clathrin-dependent endocytosis, but for cancer cells some additional mechanisms of penetration are not fully excluded. Moreover, the amount of NDs internalized is significantly more in cancer cells than in non-cancer cells. Note that for cancer cells, clathrin-dependent endocytosis was also observed previously in researches of Faklaris et al. ${ }^{27}$ Moore et al. ${ }^{32}$ used lipids to make a thin film on the NDs surface and obtained a cancer-targeting characteristic onto the liposomal complex with anti-EGFR (Epitelial Growth Factor Receptor) antibodies. In addition, functionalized NDs have been studied to enhance the efficacy of cancer drugs. Another example for targeting chemotherapy with NDs is represented by multimodal NDs particles studied by Zhang et al. ${ }^{11}$ The authors attached both a fluorescein labeled dTnucleotide for paclitaxel delivery and used an anti-EGFR monoclonal antibody on the NDs surface for selective targeting with the hetero-bifunctional crosslinker sulfosuccinimidyl 6-(30-[2-pyridyldithio]propionamido) hexanoate (sulfo-LC-SPDP). The obtained NDs selectively entered into EGFR-overexpressing MDA-MB-231 cancerous cells than in the case of basal EGFR expressing MCF7 cells. Along with visualizing the NDs, the covalent attached ND complex increased targeting and therapeutic activity as well. ${ }^{33}$ NDs can be opportunely derivatized to obtain specific tumor targeting. An example is the study carried out by Purtov et al. ${ }^{34}$ Surface of DNDs was functionalized for the covalent attachment of immunoglobulin, and simultaneously bovine serum albumin and
Rabbit Anti-Mouse Antibody. The nanodiamond-IgGI125 and RAM-nanodiamond-BSAI125 complexes are stable in blood serum and the immobilized proteins retain their biological activity. It was shown that the RAMnanodiamond-BSAI125 complex is able to bind the target antigen immobilized on the Sepharose 6B matrix through antibody-antigen interaction. The idea can be extended to use nanodiamonds as carriers for delivery of bioactive substances (i.e., drugs) to various targets in vivo. ${ }^{34}$

\section{2. $\mathrm{pH}$ Sensitive Targeting}

$\mathrm{pH}$-sensitive nanocarriers can be destabilized when the external $\mathrm{pH}$ is changed, usually from a neutral or slightly alkaline $\mathrm{pH}$ to an acidic $\mathrm{pH}$. They are designed to circumvent delivery of nanocarrier contents to the lysosomes of cells following internalization of the vesicle via the endocytic pathway ${ }^{35}$ and to release their content in particular sites of the body characterized by acidic $\mathrm{pH}$ such as: tumor and inflammation zones. The concept of $\mathrm{pH}$ sensitive nanocarriers emerged from the fact that certain enveloped viruses developed strategies to take advantage of the acidification of the endosomal lumen to infect cells, as well as from the observation that some pathological tissues (tumors, inflamed and infected areas) exhibit an acidic environment as compared to normal tissues. Different classes of $\mathrm{pH}$-sensitive nanocarriers have been proposed in the literature according to the mechanism triggering $\mathrm{pH}$-sensitivity. ${ }^{36-38}$ Guan et al. ${ }^{39}$ reported the possibility of entrap Cisdichlorodiammineplatinum(II) (CDDP, cisplatin) in $\mathrm{pH}$ sensitive NDs conjugating the carboxylic groups of the carriers with Pt(II) due to their higher affinity. Actually, numerous $\mathrm{pH}$ sensitive nanocarriers have been evaluated for CDDP delivery as well as for other anticancer drugs. However, the biocompatibility of the studied carriers still receives continued assessment, which increases adverse effects during chemotherapy. Owing to their superior chemical and physical properties as well as good biocompatibility, diamond-based materials have been attracting great attention for biological applications. Previous works have examined the use of diamond thin films produced by chemical vapor deposition (CVD) for robust implant coatings and biosensors. ${ }^{40,41}$ Herein, the authors 
demonstrate that NDs serve as an efficient CDDP vehicle. CDDP was successfully loaded onto NDs by adsorption and complexation. The $\mathrm{pH}$-controlled release properties of the carrier system were investigated comparing the release rate in different solutions at $\mathrm{pH} 6.0$ and 7.4. In addition, the CDDP released from the NDs complex retained its anticancer activity in vitro; this behavior was observed from the inhibition of proliferation of human cervical cancer (HeLa) cells. Therefore, the cited work demonstrates a new type of controlled-release system for CDDP, and suggests a strong potential for the application of NDs as significant drug-delivery nanomaterial. ${ }^{39}$ In a more recent study Yan et al. ${ }^{42}$ firstly demonstrated that doxorubicine (DOX) loaded NDs are $\mathrm{pH}$ sensitive, as the addition of a base improves drug loading, while the addition of an acid triggers drug release. Theoretical calculations were applied to further understand the release mechanism. Moreover, NDs were coupled with fluorescein to produce Fc-NDs-DOX that have promising applications in imaging as well as triggered DOX delivery, making them good candidates for theranostic applications. The use of theranostic nanoparticles, which combine both therapeutic and diagnostic capabilities, has the potential to advance the biomedical field toward personalized medicine. ${ }^{1}$ The results show that basic conditions increase the loading efficiency and acidic conditions are favorable to the release of DOX from the Fc-NDs complex. Furthermore, theoretical calculations support this conclusion since the interaction energies between NDs surface carboxyl groups and DOX at $\mathrm{pH}<7, \mathrm{pH} 7$ and $\mathrm{pH}>7$ are $10.4 \mathrm{k} \mathrm{cal} \mathrm{mol}^{-1}$, $25.0 \mathrm{k} \mathrm{cal} \mathrm{mol}^{-1}$ and $27.0 \mathrm{k} \mathrm{cal} \mathrm{mol}^{-1}$ respectively. The loading efficiency is $64 \mu \mathrm{g} \mathrm{mg}^{-1}(6.4 \mathrm{wt} \%)$ and the desorption ratio can reach to $50 \%$ at $25^{\circ} \mathrm{C}$ and $80 \%$ at $37{ }^{\circ} \mathrm{C}$ in one day. Cellular imaging experiments show that $\mathrm{Fc}$ NDs can easily enter breast adenocarcinoma (BA) cells and cell viability results prove that Fc-NDs are safe to use as drug carriers, in both neutral and acidic conditions. In addition, DOX release has been tested in vitro on BA cells, showing that the release is activated at $\mathrm{pH}$ 4.83. Thus, these Fc-NDs have a promising dual potential of imaging and controlled drug release at the same time, making them suitable for theranostic nanomedicine. ${ }^{42}$

\subsection{Cancer Therapy}

An ideal nanocarrier to be effective in cancer treatment must: enhance drug therapeutic efficacy, be able to selective target the pathological site minimizing the drug distribution throughout the body and be able to track the drug. The most recently studied nanocarriers must incorporate all these useful properties. Up to date nanocarriers have been widely studied for cancer treatment and a lot of them are on the market and commonly used. However, NDs are particularly attractive, for their solubility, low toxicity and for the absence of cellular resistance mechanism with all of the issues in one particle (Table II). In a comparative study the nanodiamond cluster interactions with several water-insoluble therapeutics was evaluated to enhance their suspension in water with preserved functionality. These therapeutics include Purvalanol A, a highly promising compound for hepatocarcinoma (liver cancer) treatment, 4-hydroxytamoxifen (4-OHT), an emerging drug for the treatment of breast cancer, as well as dexamethasone, a clinically relevant anti-inflammatory that has addressed an entire spectrum of diseases that span complications from blood and brain cancers to rheumatic and renal disorders. The authors demonstrated that NDs-drug interactions, enhanced dispersion in water, as well as preserved drug activity. That is an evidence of the broad applicability of the NDs toward delivering poorly water-soluble therapeutics to realize novel treatment routes. To assess drug functionality following NDs complexing, DNA laddering assays were performed to confirm Purvalanol A-induced DNA fragmentation demonstrating the retained biological activity of Purvalanol after undergoing sequestration to and release from the NDs. ${ }^{43}$ Additionally, the chemotherapeutic effects of the ND 4-OHT complexes were evaluated via MTT cell viability assay and no significant difference in cell viability between MCF-7 cultures with and without NDs was assessed, confirming the reported biocompatibility of NDs. Moreover, the NDs 4-OHT complexes have the same magnitude of chemotherapeutic potency as the drug alone. ${ }^{43}$ Another study coupled the NDs technology to a microfilm device. The development of a scalably fabricated flexible microfilm patch device harnesses the release activity of NDs for significantly extended drug release in a specific site. These nanodiamond-embedded polymeric microfilms utilize the aforementioned benefits associated with NDs with minimal to no impact upon the dimensions of the device, thereby realizing a high-load capacity system. Because of the innate biostability of the parylene polymer platform that was utilized for encapsulation, this technology can be useful to prepare a long termdrug elution, and viral infection in a tangible form allowing directed device placement toward virtually any specified location. The hybrid film consists of doxorubicin (DOX)-NDs conjugates located between a thick base and thin permeable layer of parylene C. NDs efficiently load DOX, and can be released gradually upon appropriate stimuli, that is, DOX concentration gradients and acidic $\mathrm{pH}$ conditions, which have been shown to be indicative of cancerous cells. The authors postulate that by altering DOX-NDs deposition amounts and the thickness of the permeable parylene layer, dosage amounts and thus, total release time can be calibrated. Since drug release is presumably driven by drug concentration gradients, the patches can be optimized to reduce elution rates until the local therapeutic concentration reach a defined threshold. Since the DOX-NDs complex is highly efficient and useful for controlled drug release, the patch may serve as a transformative technology for pre- and postoperative therapies 
Table II. NDs in cancer therapy.

\begin{tabular}{|c|c|c|c|}
\hline Composition & Drug & Results & References \\
\hline NDs & Purvalanol A, 4-hydroxytamoxifen & $\begin{array}{l}\text { NDs enhance drug dispersion in water, and mantain } \\
\text { drug cytotoxicity }\end{array}$ & [43] \\
\hline $\begin{array}{l}\text { Nanodiamond-embedded polymeric } \\
\text { microfilms }\end{array}$ & Doxorubicin & $\begin{array}{l}\text { Slow release; DOX-NDs patch in pre- and } \\
\text { postoperative therapies; localized therapeutic } \\
\text { release and tumor apoptosis prior to surgical } \\
\text { intervention, or reduce the recurrence of cancer } \\
\text { after surgery }\end{array}$ & [44] \\
\hline $\begin{array}{l}\text { Novel covalently bonded ND-PTX } \\
\text { conjugate }\end{array}$ & Paclitaxel & $\begin{array}{l}\text { Mitotic arrest and apoptosis of cancer cells. } \\
\text { Inhibition of tumorigenesis in human lung cancer } \\
\text { cells in xenograft SCID mice }\end{array}$ & [45] \\
\hline $\begin{array}{l}\text { DOX and TAT conjugated to NDs surface } \\
\text { through carbodiimide coupling }\end{array}$ & Doxorubicin & $\begin{array}{l}\text { TAT-ND-DOX enhance translocation across C6 } \\
\text { glioma cells membrane and exhibit a higher } \\
\text { cytotoxicity effect }\end{array}$ & [48] \\
\hline NDs covalently conjugated with PEG-4000 & Doxorubicin & $\begin{array}{l}\text { Delivery of Dox into HepG2 via a clathrin-dependent } \\
\text { endocytosis, increase of uptake half-life of } \\
\text { ND-PEG-DOX in comparison to free DOX. }\end{array}$ & [49] \\
\hline NDs embedded in PEG diacrylate gels & Daunorubicin and Doxorubicin & Sequential elution & {$[50]$} \\
\hline DSPE-PEG $2 \mathrm{~K}$ coating NDX DNX & Doxorubicin & $\begin{array}{l}\text { Enhancement of DOX cytotoxicity, cell apoptosis, } \\
\text { inhibition of lung metastasis of breast cancer, } \\
\text { reduction of systemic toxicity }\end{array}$ & {$[50]$} \\
\hline $\begin{array}{l}\text { ND with cisplatin incorporated via direct } \\
\text { attachment to ND surface, physical } \\
\text { adsorption POEGMEMA surface coating, } \\
\text { or complexation to MAETC groups of a } \\
\text { POEGMEMA-st-PMAETC surface layer }\end{array}$ & Cisplatin & $\begin{array}{l}\text { All ND systems displayed lower IC50 values than } \\
\text { free cisplatin in A2870 and A2870cis ovarian } \\
\text { cancer cells. POEGMEMA-coated particles the } \\
\text { most cytotoxic, }\end{array}$ & [52] \\
\hline
\end{tabular}

to induce localized therapeutic release and tumor apoptosis prior to surgical intervention, or reduce the recurrence of cancer after surgery. ${ }^{44}$ Liu et al. ${ }^{45}$ carried out a pioneristic study on the Paclitaxel (PTX) delivery by NDs. In this study, a novel covalently bonded NDs-PTX conjugate was prepared for cancer therapy. NDs-paclitaxel can be used to treat human lung carcinoma cells. Interestingly, NDs-paclitaxel displayed anticancer activities by inducing mitotic arrest and apoptosis. This is the first time to demonstrate that ND-PTX inhibits the tumorigenesis of human lung cancer cells in xenograft SCID mice but ND alone did not induce the effects on mitotic arrest, apoptosis and antitumorigenesis. ${ }^{45}$ In another study the importance of NDs interaction with cell proteins and the toxicity of NDs to three kinds of mammalian cells were evaluated. The study indicated that serum proteins in cell culture medium had significant effect. When cells were exposed to NDs dispersed in complete cell culture medium, no cytotoxicity was detected. However, severe cell death was found after exposition to NDs dispersed in serum-free medium. Possible reasons for serum-dependent cytotoxicity were discussed and the potential influence of serum on the efficacy was pointed for anticancer drug delivery system based on NDs. The present study showed that NDs were able to largely adsorb serum proteins in culture medium and the adsorbed amount of serum proteins was determined to be as high as $48 \mathrm{wt} \%$ at $37{ }^{\circ} \mathrm{C}$. After internalization, the 'bare' NDs uncoated by serum proteins were responsible for broad cell death, while, the serum proteins coated NDs seemed to play a protective role of cells. The experiments showed that serum could stabilize the dispersion of NDs in culture medium probably via increasing the hydrophilicity and reducing the size of aggregates of NDs. Then adsorption of anticancer drug 10-hydroxycamptothecin (HCPT) by NDs was studied. Experiments indicated that diluted $\mathrm{NaOH}$ solution could promote the loading of HCPT on NDs and a slow release of HCPT from the HCPT-ND complex was established in low $\mathrm{pH}$ media. Assessments of cell viability and imaging with transmission electron microscopy demonstrated a much higher efficacy of the HCPT-ND complex compared with HCPT alone. ${ }^{46}$ Several studies have been performed to study different formulations of NDs and DOX. For example, DOX and cell penetrating peptide TAT were conjugated to the surface of NDs in sequence through carbodiimide coupling in order to avoid premature release and enhance the intracellular delivery of DOX. The cell penetrating peptide TAT, ${ }^{47}$ not only show a highly efficient translocation through membranes into cytoplasm, but also circumvent the chemotherapeutic resistance in cells, increasing the cytotoxicity against cancer cells. The authors demonstrated that conjugation of TAT to ND-DOX could enhance the translocation across the C6 glioma cells membrane and exhibit a higher cytotoxicity effect than free DOX. ${ }^{48}$ In another study NDs were covalently conjugated with hydroxypolyethylene glycol-4000 (PEG-4000) and then DOX was physically adsorbed onto the PEGylated nanodiamond (ND-PEG-OH). The authors 
found that the formulation ND-PEG-DOX is able to efficiently deliver the drug into the human liver cancer cells (HepG2) via a clathrin-dependent endocytosis pathway, and especially to enhance the DOX uptake as compared to the drug alone. The uptake half-life of ND-PEG-DOX was approximately two times that of free DOX uptake. The results also demonstrated the ability of DOX to detach from ND-PEG-DOX composites inside the cytoplasm and to migrate and enter in the nucleolus to inhibit the cellular growth. ${ }^{49}$ PEG was also used in another study to form a matrix loaded with ND-drug complexes to form a multi-drug releasing tablet with sequential elution capabilities. NDs embedded in PEG diacrylate (PEGDA) gels were prepared and widely characterized. Furthermore, the addition of PEGDA has been shown to decrease degradation and improve the elastic modulus of gel materials, such as polymer tissue adhesives. The chemotherapeutics DOX and Daunorubicin (DNR), which rely on intercalation of DNA to kill cancerous cells, were used as model drugs for these release experiments, and their post release activity was verified using a cellular viability assay. A duallayered PEGDA structure with ND-drug carriers, was also prepared and demonstrated the sequential elution of DNR and Temozolomide (TMZ). ${ }^{50}$ DOX was also physically dispersed into long-circulating NDs-mediated drug delivery system with excellent dispersibility and high stability. The system was coated by 1,2-distearoyl-sn-glycero-3phosphoethanolamine- $N$-[methoxy(polyethylene glycol)2000] (DSPE-PEG 2K). The resulting system DSPE-PEG $2 \mathrm{~K}$ coating the NDs-DOX complex (NDX) was first investigated to inhibit the lung metastasis of breast cancer. DSPE-PEG 2K coated NDs-DOX significantly enhanced the cytotoxicity of DOX, induced cell apoptosis, and inhibited the lung metastasis of breast cancer with reduced systemic toxicity. In addition, NDX exhibited good tissue compatibility. ${ }^{50} \mathrm{~A}$ recent work by $\mathrm{Xi}$ et al. ${ }^{51}$ deepened the use of NDs for DOX delivery for treatment of malignant brain gliomas via convection-enhanced delivery (CED). CED is expected to overcome obstacles associated with the treatment of glioblastoma; the lack of penetration of the blood-brain barrier (BBB) and insufficient concentration of the agent in tumor tissue after systemic administration. NDs effectively bind DOX, resulting in markedly reduced myelosuppression while mediating sustained therapeutic release and enhanced efficacy. To demonstrate the applicability of the ND-DOX complex for glioblastoma treatment, uptake and retention of unmodified DOX and ND-DOX was investigated in vitro and additional parameters were investigated in vivo: toxicity and distribution in healthy rat brain, and efficacy in a rodent glioma model. The results indicate that ND-DOX administered via CED improves drug distribution and retention in brain tissue. In addition, ND-DOX increased apoptosis and decreased cell viability in glioma cell lines. In vivo results in normal rat brain tissue showed, for the first time, that ND-mediated delivery limited DOX distribution, and reduced toxicity, while increasing retention. Finally and most critically, therapeutic efficacy was enhanced and survival time increased in rats treated with ND-DOX compared to unmodified DOX. ${ }^{51}$ An interesting work of comparison between three different formulations was carried out by Huynh et al. ${ }^{52}$ They compared the cytotoxicity of three ND-cisplatin systems in which cisplatin was incorporated via direct attachment to the ND surface, physical adsorption within a poly(oligo(ethylene glycol) methyl ether methacrylate) POEGMEMA surface coating, or complexation to 1,1-di-tert-butyl 3-(2-methacryloyloxy)ethyl)butane-1,1,3tricarboxylate (MAETC) groups of a POEGMEMA-stPMAETC surface layer. All three ND systems displayed lower IC50 values than free cisplatin in ovarian cancer cells. The two polymer-containing systems out performed their 'naked' counterpart, with the POEGMEMA-coated particles the most cytotoxic, more than an order of magnitude lower than that of cisplatin. The enhanced cytotoxicity is attributed to promotion of cellular uptake by the hydrophilic surface polymer. ${ }^{52}$

\subsection{Antibacterial Use}

NDs can exploit an anti bacterial activity non only loading anti bacterial drugs, but by immobilizing bacteria on their surface. This activity is of high importance in the case of implants, where NDs loaded with opportune enzymes or polysaccharides can conjugate and remove bacteria or loaded with silver particles can reduce infections (Table III). Lysozyme for example is a globular antimicrobial protein able to lyse the bacterial cell wall by catalyzing the hydrolysis of the sulfur backbone of the peptidoglycan component thereby distorting the normal packing between the phosphate groups of the phospholipids and lipopolysaccharides. Nguyen et al. ${ }^{53}$ demonstrated that lysozyme linkage to NDs surface maintain

Table III. NDs in antibacterial use.

\begin{tabular}{|c|c|c|c|}
\hline Composition & Drug & Results & References \\
\hline $\begin{array}{l}\text { Saccharide- } \\
\text { modified NDs; } \\
\text { Glyco-NDs }\end{array}$ & $\begin{array}{l}\text { No drug, but } \\
\text { linkage with } \\
E \text {. coli } \\
\text { through } \\
\text { specific } \\
\text { protein- } \\
\text { carbohydrate } \\
\text { interactions }\end{array}$ & $\begin{array}{l}\text { NDs useful to } \\
\text { flocculate bacteria } \\
\text { and allow their } \\
\text { removal by } \\
\text { filtration }\end{array}$ & [56] \\
\hline NDs composites & $\mathrm{Ag}$ & $\begin{array}{l}\text { With low doses of } \\
\text { Ag/NDs the growth } \\
\text { of } E \text {. coli was } \\
\text { almost inhibited }\end{array}$ & {$[57]$} \\
\hline $\begin{array}{l}\text { NDs coupled with } \\
\text { an antigen able } \\
\text { to identify } \\
\text { Mycobacterium } \\
\text { tuberculosis } \\
\text { complex }\end{array}$ & $\begin{array}{l}\text { Antigen } \\
\text { captured by } \\
\text { NDs is cell } \\
\text { filtrate protein } \\
10(\text { CFP-10) }\end{array}$ & $\begin{array}{l}\text { Detection of } \\
\text { Mycobacterium } \\
\text { tuberculosis rapid, } \\
\text { specific, safe, } \\
\text { reliable, and } \\
\text { inexpensive }\end{array}$ & [58] \\
\hline
\end{tabular}


its hydrolytic activity. Then Perevedentseva et al. ${ }^{54}$ found that a formulation of NDs with lysozyme electrostatically adsorbed can bind on E. coli surface. There appeared to be no specific binding or interaction between the nonconjugated NDs and E. coli, but ND-lysozyme showed to attach and perturb the outer cell membrane resulting in significant decreases in bacterial colonies compared with controls and similar activity to lysozyme in solution. It was demonstrated that NDs loaded with sol-gel synthesized epoxy-silicate coatings have mild antimicrobial effects against seven different mold fungi. ${ }^{55}$ In the study of Hartmann et al. ${ }^{56}$ the synthesis and application of a novel, saccharide-modified ND material for the detection and agglutination of bacteria is reported. The saccharide molecule is covalently linked to ND surface. The linkage with $E$. coli is through specific protein-carbohydrate interactions. Bacterial adhesion to cell surfaces can eventually lead to the formation of biofilms and infection of the host organism. The surface decoration can be flexibly selected according to the targeted interaction, the synthesis strategy is suited for a wide range of applications. Depending on the respective surface termination the ND conjugates can either interact specifically or nonspecifically with the surface of different pathogenic bacterial strains. The agglutination experiments with $E$. coli corroborate ND particles as a useful means to flocculate bacteria and thereby allow their removal by filtration through a low-cost, highthroughput filter material with large pore size. Glyco-NDs can easily be recycled by addition of a low-cost, commercially available inhibitor of bacterial adhesion such as MeMan. ${ }^{56}$ In a recent study $\mathrm{Xu}$ et al. ${ }^{57}$ reported the synthesis and characterization of diamond-silver composite with anti-bacterial properties. The preparation of the composite has been carried out through an in situ reduction/deposition method. The obtained NDs were used as support material. They show narrow size distribution, and an uniform presence of $\mathrm{Ag}$ on their surface. The antibacterial activity of $\mathrm{Ag} / \mathrm{ND}$ composite was determined by using E. coli as test bacteria. Obtained results demonstrated that the $\mathrm{Ag} / \mathrm{ND}$ composite may have great potential as coating materials for bioimplants. ${ }^{57}$ Another aspect of the use of NDs is the possibility of use them as diagnostic method for the identification of Mycobacterium tuberculosis complex (MTBC) in broth culture media. Detonation NDs capture the antigen secreted by MTBC which is cultured in BACTEC MGIT 960 without albumin interference. The antigen captured by NDS is cell filtrate protein 10 (CFP-10), secreted in the medium of mycobacterium growth indicator tube. Five hundred consecutive clinical specimens subjected to routine mycobacteria identification in hospital were used and the sensitivity of the authors method is $100 \%$ and the specificity is 98\%. The authors demonstrated that NDs coupled with the analytical method MALDI-TOF MS for the detection of MTBC are rapid, specific, safe, reliable, and inexpensive. ${ }^{58}$

\subsection{Immunogenic Effect}

The possibility of antigen coupling to nanocarrier surface have been used to obtain an immunogenic response, but attention must be done on possible conformation alteration of the antigen that can occur after surface adsorption or shielding of critical functional groups decreasing the availability, stability, and activity of the antigen. In the study of Kossovsky et al. ${ }^{59}$ NDs have been investigated as potential antigen carriers to maintain the conformational stability of mussel adhesive protein (MAP) after injection into mice leading to antibody production. NDs with different sizes ranging in size from 5 to $10 \mathrm{~nm}$, with some 100 to $300 \mathrm{~nm}$ were coated with cellobiose, a disaccharide that was capable of hydrogenbonding to MAP to minimize surface denaturation of the adsorbed antigen. The antigen MAP-NDs complexes resulting in about $300 \mathrm{~nm}$ aggregates. The ND-MAP conjugates were intramuscularly injected into white rabbits and after 1 month the production of anti-MAP antibodies was quantified by enzyme-linked immuno sorbent assay (ELISA) demonstrating a strong and specific antibody. The success of the ND-MAP conjugate was attributed to the nature of the cellobiose matrix and protein-cellobiose interactions at the surface of the NDs. ${ }^{59}$

\subsection{Gene Therapy}

Gene therapy research is still problematic owing to a paucity of acceptable vector systems to deliver nucleic acids to patients for therapy. Viral vectors are efficient but may be dangerous for routine clinical use. Synthetic non viral vectors are fundamentally safer but are currently not efficient enough to be clinically viable. A possible solution for gene therapy lies with improved synthetic non viral vectors based upon well-established platform technologies and a thorough understanding of the barriers to efficient gene delivery and expression (transfection) relevant to clinical applications of interest. Once inside the cell, nanocarriers must offer protection from DNA degradation but do not often allow for an adequate DNA release from endosomal compartments. If gene payload is not released from endosomes, it is shuttled to the lysosomes, where it is degraded by the abundant nucleases and transfection may fail. One of the most common non viral gene delivery vectors are DNA cationic lipid complexes (lipoplexes). But to overcome this problem, researchers are lavishing many efforts to develop an efficient nanocarrier. ${ }^{60} \mathrm{NDs}$ can also serve as a safe and effective alternative to viral gene delivery. When coated with polyethyleneimine (PEI), a polycationic polymer, NDs are capable of safely and efficiently introducing DNA coding for the production of multiple proteins to the cells in a culture. ${ }^{10}$ RNA interference (RNAi) delivery is another field where NDs have a potential impact. Alhaddad et al. performed two different studies to assess the possibility to deliver nucleic material to Ewing sarcoma. In the first study NDs were 
used as a cell targeting bifunctional device: a drug-delivery vehicle and a fluorescent label at the same time. NDs were used as a cargo to deliver siRNA in human cell lines originating from Ewing sarcoma tumors. Treatment of this cancer involves surgical resection, radiotherapy, and chemotherapy. New strategies based on the inhibition of EWS-Fli1 gene by antisense oligonucleotides or siRNA targeting the EWS-Fli1 junction at the mRNA level have been developed. ${ }^{61}$ siRNA were shown to trigger the EWSFli1 mRNA cleavage and showed an inhibition in vitro of EWS-Fli1 expression and in vivo of tumor growth. Cationic NDs were developed to bind siRNAs at their surface comparing the effectiveness of two types of polymers capable of interacting with anionic oligonucleotides: polyethyleneimine (PEI) and polyallylamine hydrochloride (PAH). PEI is currently used as an oligonucleotide transfection agent. However, the water-soluble PEI does not form siRNA polyplexes stable enough in extracellular media for effective delivery, but the system consisting in non covalent coating of PEI to the surface of a nanoparticle has proved to be a very efficient DNA and siRNA delivery device. PAH forms a reproducible complex leading to a stable aqueous suspension with a low cell toxicity. Fluorescent NDs, were used to evaluate their capacity (i) to bind to siRNA, (ii) to allow its cell uptake, and (iii) to inhibit EWS-Fli1 expression in cell culture at both the mRNA and protein levels. This vector shows advantages compared to the usual transfection agent Lipofectamine, inducing a larger efficiency in inhibiting the EWS-Fli1 expression, combined with a lower toxicity to the cell in serum supplemented medium. A larger adsorption affinity of siRNA onto PAH-coated NDs than onto PEI-coated NDs was observed and results in a much slower dissociation of the siRNA:ND-PAH complex than of the siRNA:ND-PEI ones, and hence a lower siRNA-associated biological activity. Moreover, the less toxicity of ND-PEI carrier than the ND-PAH ones has been demonstrated. ${ }^{62}$ In the second study the uptake mechanisms and cellular localization of the two different types of cationic polymer coated NDs vectors were investigated. siRNA gene silencing occurred only if the siRNA:cationic nanodiamond complex followed the macropinocytosis route of internalization in the NIH/3T3 murine fibroblast cells stably transfected with the human EWS-Fli1 oncogene. The authors demonstrated that the nature of the polymer coating plays an essential role determining the siRNA:ND uptake mechanisms used and the biological activity of the siRNA. ND-PEI internalization occurred by both endocytosis and macropinocytosis, whereas ND-PAH tended to stay outside the cell, entering only by clathrin-mediated endocytosis. Despite the existence of several different pathways for cationic NDs uptake, the obtained results indicate that only the macropinocytosis pathway, which mediates the uptake of ND-PEI:siRNA, allows the efficient delivery of siRNA to the cell, resulting in destruction of the target
mRNA. By contrast, the clathrin-dependent endocytosis pathway delivers the siRNA and its carrier to the lysosomal compartment, in which siRNA hydrolysis probably occurs. ${ }^{63}$ In an interesting study Kim et al. ${ }^{64}$ developed a modeling framework to accurately guide the design of ND-PEI gene platforms and elucidate binding mechanisms between NDs, PEI, and siRNA. This is among the first NDs simulations to comprehensively account for NDs size, charge distribution, surface functionalization, and graphitization. The simulation results are compared with experimental results both for PEI loading onto NDs and for siRNA (c-Myc) loading onto ND-PEI for various mixing ratios. Remarkably, the model is able to predict loading trends and saturation limits for PEI and siRNA while confirming the essential role of ND surface functionalization in mediating ND-PEI interactions. ${ }^{64}$ Functional nanodiamonds (NDs) were also used to develop tools for DNA detection. Covalent, stable and functional conjugate of peptide nucleic acids (PNA) with $20 \mathrm{~nm}$ HPHT (High Pressure High Temperature) nanodiamonds were prepared. Peptide nucleic acid is a DNA mimic related to both peptides via its backbone and to nucleic acid via its bases. The novel functionalization route is based on an optimized amidation of ND carboxylic acid groups, to produce ND-PNA conjugates via an efficient, simple and reproducible method. The covalent binding of the ND-PNA and the loading of nucleic acid grafted onto the NDs were performed using various characterization methods. Then, ND-PNA conjugates were validated through a successful recognition of complementary DNA in a mixture, showing their efficiency toward nucleic acid detection. Moreover, ND-PNA showed absence of cyto-toxicity on A 549 cells. Such nucleic acid-functionalized nanodiamonds offer a wide range of applications as the possibility to target and to recognize DNA. ${ }^{65}$

\subsection{Protein Delivery}

In addition to nucleic acids delivery, NDs can also be used to control the release of therapeutic proteins. NDs have been non covalently functionalized with the peptide hormone insulin. The hormone insulin is used as a model for protein desorption due to its relative stability and measurable response in cellular studies. The adsorption and desorption characteristics of insulin on NDs as a platform for protein-based drugs were investigated by numerous techniques. Furthermore, the confirmation of protein functionality was confirmed using the RAW 264.7 cell line for insulin-mediated cellular recovery MTT assays. In addition, the 3T3-L1 cell line was utilized for quantitative real-time polymerase chain reaction assays for insulin-induced upregulated Insulin 1 and Granulocyte colony-stimulating factor gene expression, which are both readouts for cellular-insulin interactions. In this study the pH-dependency of protein desorption was also studied, the exposure of the ND-insulin complex to alkaline 
environments mediates the interaction between the NDs and insulin resulting in protein release. Imaging methods and adsorption/desorption assays reveal effective binding of insulin to the NDs and significant insulin release under alkaline conditions. MTT and RT-PCR analysis indicate preserved function following desorption, while adsorbed insulin remained largely inactive. ${ }^{66}$ In a recent study bone morphogenetic proteins (BMPs) were loaded to NDs for bone formation. These proteins are regulators of cartilage and bone development. Of the BMPs, rhBMP-2 and rhBMP-7 have been granted Food and Drug Administration approval and are used for a variety of on- and off-label procedures involving the promotion of bone formation ${ }^{67,68}$ and in oral and maxillofacial procedures (i.e., InFUSE ${ }^{\circledR}$ for use in sinus and localized alveolar ridge augmentation). The effects of BMP-2 in vivo are strictly dependent on its local concentration and this can be achieved using a carrier to prevent rapid clearance by diffusion. The potential of NDs suspensions as a protein delivery vehicle for the promotion of bone formation was evaluated by Moore et al. ${ }^{69}$ Although BMP-2 and -7 are effective at promoting bone formation on their own, there are numerous proteins involved in the signaling cascade that may augment the response to BMPs, for example the fibroblast growth factor (FGF) that is able to promote myoblast proliferation and to inhibit differentiation and at low doses to enhance BMP-2 activity in vivo. The authors demonstrate that NDs are capable of simultaneously delivering 2 functional proteins: BMP-2 and bFGF. ND-BMP induced differentiation of $\mathrm{C} 2 \mathrm{C} 12$ myoblasts into alkaline-phosphataseproducing osteo-blasts, while the combination of BMP-2 and bFGF in ND clusters induced proliferation of the precursor myoblasts in addition to differentiation. However, the response to BMP-2 and FGF in cultured cells is complex and it seems that bFGF induced proliferation while inhibiting myoblast differentiation. In contrast, in vivo the addition of FGF augments bone formation in response to BMP-2. Although the BMP-2 and bFGF pathways are competitive in cultured cells, both pathways are necessary for effective bone healing in vivo. bFGF (FGF-2) is critical for promoting the proliferation of osteoblast progenitors, while BMP-2 is necessary for stimulating osteoblast differentiation and mineral production NDs are capable of simultaneously delivering various doses of two proteins, BMP-2 and bFGF, for the promotion of bone cell differentiation and proliferation in vitro. ${ }^{69}$

\subsection{Bone Implants}

Tissue engineering is a promising technique for repairing or replacing partial or whole diseased tissues using artificial constructs. A proper scaffold must exhibit a mechanical strength similar to natural bone, good biocompatibility, and adjustable biodegradability so that the scaffold could be gradually replaced with the new growing tissue. Carbon based nanomaterials, such as singlewalled or multi-walled carbon nanotubes (SWCNTs or
MWCNTs, respectively) or NDs, have been widely used as nanofillers in polymeric films or fibers to improve the mechanical properties (i.e., elastic modulus and hardness) of the nanocomposites. ${ }^{70-74}$ A multifunctional fluorescent composite bone scaffold material has been produced utilizing a biodegradable polymer, poly(L-lactic acid) (PLLA), and octadecylamine-functionalized nanodiamond (ND-ODA). ${ }^{12}$ The uniform dispersion of nanoparticles in the polymer led to significant increase in hardness and Young's modulus of the composites. Addition of $10 \% \mathrm{wt}$ of ND-ODA resulted in more than 200\% increase in Young's modulus and $800 \%$ increase in hardness, bringing the nanocomposite properties close to that of the human cortical bone. ND-ODA/PLLA were also studied as a matrix supporting murine osteoblast (7F2) cell growth for up to 1 week and the addition of ND-ODA had no negative effects on cell proliferation. ND-ODA can act as a multifunctional nanocarrier characterized by improved mechanical properties, bright fluorescence, and useful for drug loading and delivery via surface modification. Cytotoxicity and biocompatibility experiments together with osteogenic marker gene expression analysis demonstrated that both NDs and ND-ODA are nontoxic to murine osteoblasts, and support cell proliferation and differentiation in vitro. ${ }^{12}$ A different approach is the one proposed by Mansoorianfar et al. ${ }^{75}$ who prepared diamond nanoparticles in alginatebioactive glass films by electrophoretic process as functional coatings for biomedical implants. The characteristics of the films were analyzed and the effect of NDs on the in vitro bioactivity and cell toxicity was examined. The results indicate that NDs hamper co-deposition of alginate and $\mathrm{BG}$ during EPD and that the addition of BG particles enhances the deposition kinetics of alginate while NDs reduces the deposition rate. The deposited alginate film showed a rough surface due to the gas bubbles. The addition of BG particles increased the surface roughness while NDs showed no effect. In vitro bioactivity assay revealed the formation of granular HA particles on the surface of the composite films after 28 days of incubation. The particles were cauliflower-like with an average size of about $70 \mathrm{~nm}$. The presence of NDs enhanced formation of HA without a change in the morphology, size, and composition of the apatite particles. MTT assay using human osteoblast-like cells showed an improved cell viability of the coated substrates due to appropriate surface nanotopography and changes in the wettability and zeta potential. $^{69}$

\subsection{Imaging}

The presence of NDs is biological systems can be detected using different techniques, taking advantage of intrinsic properties as well as properties obtained through different modifications/functionalizations of NDs. Therefore, in addition to their visualization to understand their fate in biological tissues, NDs have been used as biomarkers, e.g., in intracellular labelling or in vivo tracking and imaging, 
also due to the relative easiness of specifically functionalizing their surface. Transmission electron microscopy (TEM) and high resolution TEM (HRTEM) have been used by Zurbuchen et al. ${ }^{76}$ to localize single ND particles in HeLa cells with high spatial resolution revealing their adherence on the nuclear membrane, while both the crystalline fringes visualized in the real space and the electron diffraction pattern confirmed the crystallinity of ND particles. TEM has been used to investigate the uptake of functionalized NDs into different biological tissues, e.g., Ewing sarcoma ${ }^{63} \mathrm{HeLa},{ }^{46,77}$ and human breast adenocarcinoma cells. ${ }^{42}$ Finally, scanning electron microscopy (SEM) has been used to study the interaction of NDs with the surface of E. coli cells. ${ }^{56}$

While NDs with ideal crystalline structure are not fluorescent or optically absorptive in the near-infrared (NIR) ${ }^{78}$ they are made fluorescent by the presence of color centers, mainly nitrogen vacancies. ${ }^{17,18,20}$ In addition, fluorescence can be induced in NDs by the covalent conjugation to the NDs surface of specific molecules, such as octadecylamine. ${ }^{19,79}$ Fluorescent NDs have been proposed as ideal biomarkers for long term imaging, as they are high photostable and do not photobleach or blink on relatively long time scales. ${ }^{20}$ Due to these unique properties, fluorescent NDs have been used as contrast agent in several studies to visualize internalization pathways and cellular uptake of several molecules in different biological tissues. ${ }^{11,31,45,63}$ Taking advantage of the time stability of fluorescent NDs, Kuo et al. ${ }^{21}$ used them as probes to investigate the intracellular transport and the of yolk lipoproteins in a nematode (Caenorhabditis elegans) using fluorescence lifetime imaging microscopy. Also, as nitrogen vacancies are located in the inner of NDs particles, the resulting fluorescence is not altered by surface functionalizations. ${ }^{20}$ In addition, in a recent work Zhang et al. $^{80}$ demonstrated the synthesis of water dispersible NDs with tunable photoluminescence. Finally, core-shell multifunctional nanocomposites have been obtained by incorporating fluorescent NDs cores in silica shells. ${ }^{81-83}$

ND exhibits a sharp Raman peak approximately at $1330 \mathrm{~cm}^{-15}$ which is generally well distinguished from Raman signals from biological samples. ${ }^{17,20,31}$ Therefore, NDs can be detected in biological systems by mapping the intensity of such a characteristic Raman peak on the sample with a spatial resolution that can be as small as $100 \mathrm{~nm}$. Raman mapping has been used to study the internalization of NDs into different cells, i.e., A549 lung human adenocarcinoma, Beas- $2 \mathrm{~b}$ non-tumorigenic human bronchial epithelial, and HFL-1 fibroblast-like human fetal lung cells. ${ }^{31}$ Here, we would mention that although atomic force microscopy (AFM) has been used to study the size distribution of NDs on substrates ${ }^{45,56}$ and to visualize NDs interacting with cells surfaces,${ }^{29}$ it could be used to obtain Raman mapping at the nanoscale through an AFM based technique, namely the tip-enhanced Raman spectroscopy
(TERS). To the best of our knowledge, such a promising new approach has not been attempted yet.

In a recent work, Kucsko et al. ${ }^{84}$ have demonstrated an exciting new approach in which electron spin resonance (ESR) spectroscopy of nitrogen vacancy color centers in NDs is used to measure the local environment at submicrometrical scale. Moreover, the combination of gold nanoparticles (to locally heat the sample) and NDs (to measure the temperature gradient) allowed the authors to control and map temperature variations at the subcellular scale in single human embryonic fibroblasts without compromising their viability.

Recently Zhang et al. $^{78}$ reported on the use of $\mathrm{He}^{+}$ion beam irradiation to obtain detonation NDs with high NIR absorption. The authors used such NDs as contrast agent for photoacoustic imaging, where a pulsed NIR laser is used to produce a periodical local heating of the sample, leading to the emission of ultrasonic waves which are collected by a transducer to reconstruct the three dimensional image of the sample. The authors demonstrated the potential of radiation damaged NDs as photoacoustic contrast agent both ex vivo in raw chicken breast and in vivo in mice.

Finally, in vivo distribution of functionalized NDs has been imaged through the conjugation to various radionuclides. ${ }^{85}$ Rojas et al. ${ }^{86}$ studied the in vivo biodistribution of NDs labelled with ${ }^{18} \mathrm{~F}$ radionuclide in mice using positron emission tomography (PET), also after the addition of either Tween 80 or PEG-8000 to the labelled NDs. Finally, Manus et al. ${ }^{8}$ reported the about tenfold increase in the contrast in magnetic resonance imaging (MRI) of NDs conjugated to $\mathrm{Gd}(\mathrm{III})$, which makes $\mathrm{Gd}(\mathrm{III})-\mathrm{ND}$ a promising contrast agent for MRI.

\subsection{Toxicological Aspects}

Carbon-based materials generally have been considered chemically inert with minimal reactivity toward cells of the body. However, carbon nanocarriers (i.e., nanotubes, fullerenes, nanodiamonds), with very small sizes, high surface to volume ratios, and reactive surface chemistries, show high cellular permeability and dynamics. ${ }^{15,87}$ The carbon nanocarrier biocompatibility, rely on the elemental impurities and structure (i.e., ropes, aggregates) of the final product. Some examples of impurities include metallic species, $\mathrm{Fe}$ and transition metals or metal residues, ceramic graphitic or amorphous material and so on depending on the preparation method. Among all carbon nanocarriers, NDs show good biocompatibility.

In particular Zhang et al. ${ }^{77}$ carried out a comparative study of cellular uptake and cytotoxicity of multi-walled carbon nanotubes (MWNT), graphene oxide (GO), and nanodiamond. They demonstrated that all the nanomaterials were internalized by HeLa cells through a non specific cellular uptake and that ND were taken up more than MWNT and GO and that this is related their structural 
characteristics. The key biological responses induced by these three systems were evaluated by biological assays, including the 3-(4,5-dimethylthiazol-2-yl)-2,5-diphenyltetrazolium bromide, malondialdehyde, superoxide dismutase, lactate dehydrogenase and reactive oxygen species. Citotoxicity studies evidenced a concentration dependent effect and that NDs show the higher internalization ratio with the lower toxicity. ${ }^{77}$ Data concerning NDs toxicology in literature are conflicting, usually they show high biocompatibility with selected cellular systems in vitro and NDs-mediated systemic toxicity in vivo. Therefore further comprehensive studies on NDs effects on other biological systems are required. For example Dworak et al. ${ }^{88}$ investigated the potential genotoxic and mutagenic activity of commercially available diamond nanopowder (particle size $<10 \mathrm{~nm}$ ) in human peripheral lymphocytes in vitro. They chose human peripheral lymphocytes, because they represent the first exposed cell types upon intravenous administration of NDs in therapeutic and diagnostic nanodevices. They checked the effect of NDs after long-term treatment (72 h) and found that NDs cytotoxicity was evidently manifested at concentration of $50 \mu \mathrm{g} / \mathrm{ml}$ and was potentiated at concentration of $100 \mu \mathrm{g} / \mathrm{ml}$. There are contrast results in the case of neuroblastoma cells that were not affected by NDs (particle size $2-10 \mathrm{~nm}$ ), while macrophages exhibited a concentration-dependent and statistically significant sensitivity to 24-h treatment of 50 and $100 \mu \mathrm{g} / \mathrm{ml}$ (MTT assay), in fact NDs may contribute to elevation in the total ROS level as well as superoxide production (both total and mitochondrial) in human lymphocytes. Moreover NDs at non-cytotoxic concentrations $(<50 \mu \mathrm{g} / \mathrm{ml})$, were able to promote genotoxicity in human peripheral lymphocytes. NDs induced DNA single-strand breaks at lowest concentration examined $(1 \mu \mathrm{g} / \mathrm{ml})$ and oxidative DNA damage and micronuclei formation at concentration of $\mu \mathrm{g} / \mathrm{ml}$. Oxidized nanodiamonds induced more potent DNA damage than raw NDs, this suggested that nanodiamond genotoxicity is surface chemistry-specific and that the NDs-induced DNA damage was mediated by oxidative stress: total ROS and specific superoxide production was elevated after NDs treatment. ${ }^{88}$ The influence of surface derivatization on NDs was also evaluated in terms of short-term cytotoxicity and embryonic development in zebrafish. In this study, the authors show that the short term cytotoxicity of a nanomaterial is determined by the surface functionality, rather than the core nanomaterial. A so-called 'non-toxic and biocompatible' nanomaterial, such as core/shell iron-filled carbon nanoparticles (FeCNPs) and NDs, can become cytotoxic when a cationic surface functionality, such as imidazolium (IM) and tertiary methyl ammonium ethyl methacrylate (TMAEA) moieties, was grafted onto the surface. The two nanomaterials FeCNPs and NDs were surface-modified with different surface functionalities: anionic $\mathrm{COOH}$, zwitterionic PVP, neutral $\mathrm{OH}$, cationic IM and TMAEA, and investigated for their cytotoxicities in both in vitro cancer cells (HeLa and U-87MG cells) and in vivo embryo development of zebrafish. Cationic IM and TMAEA functionalities of both FeCNPs and NDs cause acute cytotoxicity to a similar extent in the in vitro cancer cell experiments, as well as affect severely the embryonic development and survival rates of zebrafish. Other surface functionalities do not show particularly strong cytotoxicities. To evaluate the origins of cytotoxicities, parameters such as the lactate dehydrogenase levels, cellular ROS generation, apoptosis, and changes in lysosomal membrane integrity, mitochondrial membrane potential, the intracellular $\mathrm{pH}$, and cell cycles were evaluated. The obtained results clearly point out that surface functionality, rather than the core nanomaterials, plays a critical role in dictating the shortterm cytotoxicities. ${ }^{89}$ The above mentioned results show the necessity to deepen the toxicological aspects of NDs administration both in vivo and in vitro.

The influence of physical-chemical aspects of NDs toxicity has been demonstrated and must be more and more evaluated both in vivo and in vitro. Long-term effects of cell-internalized NDs and subsequent cell fate (e.g., potential oxidative stress or cell death) or distribution and toxicity in animal models with a particular attention to route of administration or exposure must be evaluated.

\section{NDs BASED PATENTS, PRECLINICAL AND CLINICAL TRIALS}

In spite of the large number of publications and the wide research carried out over the last few decades on NDs formulations and possible applications, as above reported, only few studies have stepped into patents and pre-clinical and clinical trials, and these are exclusively oriented towards synthetic and functionalization methods, few on the possibility to bound drugs. The 'NDs approach' was proposed for different drugs: adriamicine, ${ }^{90}$ for unspecified antitumor drugs, for cosmetic purpose. ${ }^{91}$ An interesting and recent paper is the one related to NDs use as drugs generating free radicals for treating tumors. The invention is based on generating free radicals on the surface of the nanodiamonds when they are exposed to radiation, for example ionizing radiation. In order to increase the effectiveness of the nanodiamonds, they can be complexed with a radiosensitizing agent. ${ }^{92}$ Another one is about the preparation of a block of carbon composite-nanodiamond graphite-like carbon with diamond content of 50-95 wt\% and the porosity of $40-75 \mathrm{vol} \%$ to entrap the drug. This system may be used as targeted drug delivery system into the central nervous system of a living body. That is ensured by making a surgical approach to a segment of the central nervous system specified for local administration of the drug. ${ }^{93}$ The derivitizing patents are about the possibility to obtain particles being modified by chlorine with a chlorine content of up to 14 at.\%, and to a method to link them drugs or biologically active agents. ${ }^{94}$ In another study drugs were coupled to functional groups on the surfaces 
of nanosized diamond enhance their efficacy. The method involves hydrating a plurality of NDs having a plurality of carbon chain surface molecules on its surface. Cations are embedded within the lattice structure of the NDs surface. The embedded cations attract anions that cause crystal growth. The anion are represented by drug molecules. The efficacy of antimicrobial drugs, as well as other drugs are enhanced by their attachment to the NDs. NDs were hydrated and functionalized with oxone or with silver ions from silver nitrate. A $1.5 \mathrm{~mm}$ thick polyurethane foam was created containing almost $4 \%$ of antimicrobial NDs. The foam was used in the treatment of wounds. ${ }^{95}$

No clinical trials are in progress at the moment.

\section{CONCLUDING REMARKS AND PERSPECTIVES}

NDs have been extensively studied as an alternative to carbon nantubes and they have some advantages over carbon nanotubes such as lower toxicity. As with carbon nanotubes, the in vitro/in vivo properties of NDs depend both on the surface composition and on method of their production. NDs drug delivery has not yet made its clinical debut even though these systems have been demonstrated to yield promising therapeutics. Although numerous papers, among those reported in this review, show data related to cytotoxicity of NDs and up to date there are some specific wide studies aimed to investigate toxicity of NDs after in vivo administration, in particular in long term trials. From published information it is possible to evaluate the safe concentration of NDs that can be used in cell interaction studies. The need of more specific toxicological study can represent an intriguing opportunity for toxicologists to enrich the knowledge in drug delivery system tolerability. For example there are no studies on the fate of NDs in cells, animals, humans, while the understanding of their metabolism is a fundamental step in reaching the clinical trials. We can look forward to many more efforts in developing NDs, according to the evidence that, despite the many promising proof of concept studies there is still a long road ahead to becoming a clinical reality. In addition, the big chance to the field consists of its highly interdisciplinary nature from basic chemistry and physic to clinical evaluations. This means there are still many open challenges that need to be overcome and there are still opportunities for academic and industrial scientists to make a decisive impact.

\section{References and Notes}

1. C. Marianecci, F. Rinaldi, C. Ingallina, D. Passeri, A. Sorbo, M. Rossi, and M. Carafa, Nuovo Cimento C 36, 103 (2013).

2. C. Marianecci, L. D. Marzio, F. Rinaldi, C. Celia, D. Paolino, F. Alhaique, S. Esposito, and M. Carafa, Adv. Colloid. Interfac. 205C, 187 (2014).

3. V. Danilenko, Phys. Solid State 46, 595 (2004).

4. E. Osawa, Nanodiamonds: Applications in Biology and Medicine, edited by D. Ho, Springer, New York City (2010), pp. 1-33.
5. V. N. Mochalin, O. Shenderova, D. Ho, and Y. Gogotsi, Nat. Nanotechnol. 7, 11 (2012).

6. G. Balasubramanian, I. Y. Chan, R. Kolesov, M. Al-Hmoud, J. Tisler, C. Shin, C. Kim, A. Wojcik, P. R. Hemmer, A. Krueger, T. Hanke, A. Leitenstorfer, R. Bratschitsch, F. Jelezko, and J. Wrachtrup, Nature 455, 648 (2008).

7. X.-Q. Zhang, M. Chen, R. Lam, X. Xu, E. Osawa, and D. Ho, ACS Nano 3, 2609 (2009).

8. L. M. Manus, D. J. Mastarone, E. A. Waters, X.-Q. Zhang, E. A. Schultz-Sikma, K. W. MacRenaris, D. Ho, and T. J. Meade, Nano Lett. 10, 484 (2010).

9. L. Wei, W. Zhang, H. Lu, and P. Yang, Talanta 80, 1298 (2010).

10. E. K. Chow, X.-Q. Zhang, M. Chen, R. Lam, E. Robinson, H. Huang, D. Schaffer, E. Osawa, A. Goga, and D. Ho, Sci. Transl. Med. 3, 73 ra21 (2011).

11. X. Q. Zhang, R. Lam, X. Xu, E. K. Chow, H. J. Kim, and D. Ho, Adv. Mater. 23, 4770 (2011).

12. Q. Zhang, V. N. Mochalin, I. Neitzel, I. Y. Knoke, J. Han, C. A. Klug, J. G. Zhou, P. I. Lelkes, and Y. Gogotsi, Biomaterials 32, 87 (2011).

13. L. K. Moore, M. Gatica, E. K. Chow, and D. Ho, Disruptive Science and Technology 1, 54 (2012).

14. O. A. Shenderova and G. McGuire, Ultrananocrystalline Diamond: Synthesis, Properties, and Applications, edited by O. A. Shenderova and D. M. Gruen, William Andrew, New York, NY (2006), pp. 79-114.

15. R. H. Hurt, M. Monthioux, and A. Kane, Carbon 44, 1028 (2006).

16. A. M. Schrand, S. A. Ciftan Hens, and O. A. Shenderova, Crit. Rev. Solid State 34, 18 (2009).

17. K. M. El-Say, Journal of Applied Pharmaceutical Science 1, 29 (2011).

18. J. M. Say, C. Vreden, D. J. Reilly, L. J. Brown, J. R. Rabeau, and N. J. C. King, Biophys. Rev. 3, 171 (2011).

19. H. B. Man and D. Ho, Phys. Status Solidi A 209, 1609 (2012).

20. R. Kaur and I. Badea, Int. J. Nanomed. 8, 203 (2013).

21. Y. Kuo, T. Y. Hsu, Y. C. Wu, and H. C. Chang, Biomaterials 34,8352 (2013).

22. B.-M. Chang, H.-H. Lin, L.-J. Su, W.-D. Lin, R.-J. Lin, Y.-K. Tzeng, R. T. Lee, Y. C. Lee, A. L. Yu, and H.-C. Chang, Adv. Funct. Mater. 23, 5737 (2013).

23. H. Hillaireau and P. Couvreur, Cell. Mol. Life Sci. 66, 2873 (2009).

24. A. M. Schrand, H. Huang, C. Carlson, J. J. Schlager, E. Osawa, S. M. Hussain, and L. Dai, J. Phys. Chem. B 111, 2 (2007).

25. K.-K. Liu, C.-L. Cheng, C.-C. Chang, and J.-I. Chao, Nanotechnology 18,325102 (2007).

26. O. Faklaris, D. Garrot, V. Joshi, F. Druon, J.-P. Boudou, T. Sauvage, P. Georges, P. A. Curmi, and F. Treussart, Small 4, 2236 (2008).

27. O. Faklaris, V. Joshi, T. Irinopoulou, P. Tauc, M. Sennour, H. Girard, C. Gesset, J.-C. Arnault, A. Thorel, J.-P. Boudou, P. A. Curmi, and F. Treussart, ACS Nano 3, 3955 (2009).

28. T.-L. Wee, Y.-W. Mau, C.-Y. Fang, H.-L. Hsu, C.-C. Han, and H.-C. Chang, Diam. Relat. Mater. 18, 567 (2009).

29. K.-K. Liu, C.-C. Wang, C.-L. Cheng, and J.-I. Chao, Biomaterials 30, 4249 (2009).

30. J. Rejman, V. Oberle, I. S. Zuhorn, and D. Hoekstra, Biochem. J. 377,159 (2004).

31. E. Perevedentseva, S.-F. Hong, K.-J. Huang, I.-T. Chiang, C.-Y. Lee, Y.-T. Tseng, and C.-L. Cheng, J. Nanopart. Res. 15, 1834 (2013).

32. L. Moore, E. K.-H. Chow, E. Osawa, J. M. Bishop, and D. Ho, Adv. Mater. 25, 3532 (2013).

33. D.-J. Lim, M. Sim, L. Oh, K. Lim, and H. Park, Arch. Pharm. Res. 37, 43 (2014).

34. K. V. Purtov, A. I. Petunin, A. E. Burov, A. P. Puzyr, and V. S. Bondar, Nanoscale Res. Lett. 5, 631 (2010).

35. V. P. Torchilin, F. Zhou, and L. Huang, J. Liposome Res. 3, 201 (1993). 
36. V. P. Torchilin, Adv. Drug Deliver. Rev. 64, 302 (2012).

37. M. Carafa, L. Di Marzio, C. Marianecci, B. Cinque, G. Lucania, K. Kajiwara, M. G. Cifone, and E. Santucci, Eur. J. Pharm. Sci. 28, 385 (2006)

38. L. Di Marzio, C. Marianecci, M. Petrone, F. Rinaldi, and M. Carafa, Colloid. Surface. B 82, 18 (2011).

39. B. Guan, F. Zou, and J. Zhi, Small 6, 1514 (2010)

40. A. Hartl, E. Schmich, J. A. Garrido, J. Hernando, S. C. R. Catharino, S. Walter, P. Feulner, A. Kromka, D. Steinmuller, and M. Stutzmann, Nat. Mater. 3, 736 (2004).

41. D. M. Gruen, Annu. Rev. Mater. Sci. 29, 211 (1999).

42. J. Yan, Y. Guo, A. Altawashi, B. Moosa, S. Lecommandoux, and N. M. Khashab, New J. Chem. 36, 1479 (2012).

43. M. Chen, E. D. Pierstorff, R. Lam, S.-Y. Li, H. Huang, E. Osawa, and D. Ho, ACS Nano 3, 2016 (2009).

44. R. Lam, M. Chen, E. Pierstorff, H. Huang, E. Osawa, and D. Ho, ACS Nano 2, 2095 (2008).

45. K. K. Liu, W. W. Zheng, C. C. Wang, Y. C. Chiu, C. L. Cheng, Y. S. Lo, C. Chen, and J. I. Chao, Nanotechnology 21, 315106 (2010).

46. J. Li, Y. Zhu, W. Li, X. Zhang, Y. Peng, and Q. Huang, Biomaterials 31, 8410 (2010).

47. J. P. Norman, S. W. Perry, K. A. Kasischke, D. J. Volsky, and H. A. Gelbard, J. Immunol. 178, 869 (2007).

48. X. Li, J. Shao, Y. Qin, C. Shao, T. Zheng, and L. Ye, J. Mater. Chem. 21, 7966 (2011)

49. D. Wang, Y. Tong, Y. Li, Z. Tian, R. Cao, and B. Yang, Diam. Relat. Mater. 36, 26 (2013).

50. J. Xiao, X. Duan, Q. Yin, Z. Zhang, H. Yu, and Y. Li, Biomaterials 34, 9648 (2013).

51. G. Xi, E. Robinson, B. Mania-Farnell, E. F. Vanin, K.-W. Shim, T. Takao, E. V. Allender, C. S. Mayanil, M. B. Soares, D. Ho, and T. Tomita, Nanomedicine 10, 381 (2014).

52. V. T. Huynh, S. Pearson, J.-M. Noy, A. Abboud, R. H. Utama, H. Lu, and M. H. Stenzel, ACS Macro Lett. 2, 246 (2013).

53. T.-T.-B. Nguyen, H.-C. Chang, and V. W.-K. Wu, Diam. Relat. Mater. 16, 872 (2007).

54. E. Perevedentseva, C.-Y. Cheng, P.-H. Chung, J.-S. Tu, Y.-H. Hsieh, and C.-L. Cheng, Nanotechnology 18, 315102 (2007).

55. A. I. Bojkova, O. A. Shilova, E. V. Golikova, N. I. Nikolajchuk, T. V. Khamova, S. V. Hashkovskij, D. Y. Vlasov, O. V. Frank-Kamenetsky, and V. Dolmatov, Nanotechnology International Forum/Rusanotech '08, Abstracts, V. 1M, RUSNANO, Moscow, Russia (2008), pp. 488-489.

56. M. Hartmann, P. Betz, Y. Sun, S. N. Gorb, T. K. Lindhorst, and A. Krueger, Chem. Eur. J. 18, 6485 (2012).

57. T. Xu, L. Wu, Y. Yu, W. Li, and J. Zhi, Mater. Lett. 114, 92 (2014).

58. P.-C. Soo, C.-J. Kung, Y.-T. Horng, K.-C. Chang, J.-J. Lee, and W.-P. Peng, Anal. Chem. 84, 7972 (2012)

59. N. Kossovsky, A. Gelman, H. J. Hnatyszyn, S. Rajguru, R. L. Garrell, S. Torbati, S. S. F. Freitas, and G.-M. Chow, Bioconjug. Chem. 6, 507 (1995).

60. G. Caracciolo, D. Pozzi, A. L. Capriotti, C. Marianecci, M. Carafa, C. Marchini, M. Montani, A. Amici, H. Amenitsch, M. A. Digman, E. Gratton, S. S. Sanchez, and A. Laganà, J. Med. Chem. 54, 4160 (2011).

61. A.-L. Ramon, J.-R. Bertrand, and C. Malvy, Tumori 94, 254 (2008).

62. A. Alhaddad, M.-P. Adam, J. Botsoa, G. Dantelle, S. Perruchas, T. Gacoin, C. Mansuy, S. Lavielle, C. Malvy, F. Treussart, and J.-R. Bertrand, Small 7, 3087 (2011).

63. A. Alhaddad, C. Durieu, G. Dantelle, E. Le Cam, C. Malvy, F. Treussart, and J. R. Bertrand, PLoS One 7, e52207 (2012).

64. H. Kim, H. B. Man, B. Saha, A. M. Kopacz, O.-S. Lee, G. C. Schatz, D. Ho, and W. K. Liu, J. Phys. Chem. Lett. 3, 3791 (2012).

65. C. Gaillard, H. A. Girard, C. Falck, V. Paget, V. Simic, N. Ugolin, P. Bergonzo, S. Chevillard, and J. C. Arnault, RSC Adv. 4, 3566 (2014).
66. R. A. Shimkunas, E. Robinson, R. Lam, S. Lu, X. Xu, X.-Q. Zhang, H. Huang, E. Osawa, and D. Ho, Biomaterials 30, 5720 (2009).

67. S. Govender, C. Csimma, H. K. Genant, A. Valentin-Opran, Y. Amit, R. Arbel, H. Aro, D. Atar, M. Bishay, M. G. Borner, P. Chiron, P. Choong, J. Cinats, B. Courtenay, R. Feibel, B. Geulette, C. Gravel, N. Haas, M. Raschke, E. Hammacher, D. van der Velde, P. Hardy, M. Holt, C. Josten, R. L. Ketterl, B. Lindeque, G. Lob, H. Mathevon, G. McCoy, D. Marsh, R. Miller, E. Munting, S. Oevre, L. Nordsletten, A. Patel, A. Pohl, W. Rennie, P. Reynders, P. M. Rommens, J. Rondia, W. C. Rossouw, P. J. Daneel, S. Ruff, A. Ruter, S. Santavirta, T. A. Schildhauer, C. Gekle, R. Schnettler, D. Segal, H. Seiler, R. B. Snowdowne, J. Stapert, G. Taglang, R. Verdonk, L. Vogels, A. Weckbach, A. Wentzensen, and T. Wisniewski, J. Bone Joint Surg. Am. 84, 2123 (2002).

68. K. L. Ong, M. L. Villarraga, E. Lau, L. Y. Carreon, S. M. Kurtz, and S. D. Glassman, Spine (Phila Pa 1976) 35, 1794 (2010).

69. L. Moore, M. Gatica, H. Kim, E. Osawa, and D. Ho, J. Dent. Res. 92, 976 (2013).

70. Z. Spitalsky, D. Tasis, K. Papagelis, and C. Galiotis, Prog. Polym. Sci. 35, 357 (2010).

71. R. Sengupta, M. Bhattacharya, S. Bandyopadhyay, and A. K. Bhowmick, Prog. Polym. Sci. 36, 638 (2011).

72. K. D. Behler, A. Stravato, V. Mochalin, G. Korneva, G. Yushin, and Y. Gogotsi, ACS Nano 3, 363 (2009).

73. D. Passeri, M. Rossi, A. Alippi, A. Bettucci, M. L. Terranova, E. Tamburri, and F. Toschi, Physica E 40, 2419 (2008).

74. D. Passeri, A. Biagioni, M. Rossi, E. Tamburri, and M. L. Terranova, Eur. Polym. J. 49, 991 (2013).

75. M. Mansoorianfar, M. A. Shokrgozar, M. Mehrjoo, E. Tamjid, and A. Simchi, Diam. Relat. Mater. 40, 107 (2013).

76. M. A. Zurbuchen, M. P. Lake, S. A. Kohan, B. Leung, and L. S. Bouchard, Sci. Rep. 3, 2668 (2013).

77. X. Zhang, W. Hu, J. Li, L. Tao, and Y. Wei, Toxicol. Res. 1, 62 (2012).

78. T. Zhang, H. Cui, C. Y. Fang, L. J. Su, S. Ren, H. C. Chang, X. Yang, and M. L. Forrest, J. Biomed. Opt. 18, 26018 (2013).

79. V. N. Mochalin and Y. Gogotsi, J. Am. Chem. Soc. 131, 4594 (2009).

80. X. Zhang, S. Wang, C. Zhu, M. Liu, Y. Ji, L. Feng, L. Tao, and Y. Wei, J. Colloid Interface Sci. 1, 39 (2013).

81. A. Bumb, S. K. Sarkar, N. Billington, M. W. Brechbiel, and K. C. Neuman, J. Am. Chem. Soc. 135, 7815 (2013).

82. E. von Haartman, H. Jiang, A. A. Khomich, J. Zhang, S. A. Burikov, T. A. Dolenko, J. Ruokolainen, H. Gu, O. A. Shenderova, I. I. Vlasov, and J. M. Rosenholm, J. Mater. Chem. B 1, 2358 (2013).

83. N. Prabhakar, T. Nareoja, E. von Haartman, D. S. Karaman, H. Jiang, S. Koho, T. A. Dolenko, P. E. Hanninen, D. I. Vlasov, V. G. Ralchenko, S. Hosomi, I. I. Vlasov, C. Sahlgren, and J. M. Rosenholm, Nanoscale 5, 3713 (2013).

84. G. Kucsko, P. C. Maurer, N. Y. Yao, M. Kubo, H. J. Noh, P. K. Lo, H. Park, and M. D. Lukin, Nature 200, 54 (2013).

85. Y. Zhu, J. Li, W. Li, Y. Zhang, X. Yang, N. Chen, Y. Sun, Y. Zhao, C. Fan, and Q. Huang, Theranostics 2, 302 (2012).

86. S. Rojas, J. D. Gispert, R. Martin, S. Abad, C. Menchon, D. Pareto, V. M. Victor, M. Alvaro, H. Garcia, and J. R. Herance, ACS Nano 5,5552 (2011).

87. L. Dai (ed.), Carbon Nanotechnology: Recent Developments in Chemistry, Physics, Materials Science and Device Applications, Elsevier, Amsterdam (2006).

88. N. Dworak, M. Wnuk, J. Zebrowski, G. Bartosz, and A. Lewinska, Carbon 68, 763 (2014)

89. R. Vankayala, P. Kalluru, H.-H. Tsai, C.-S. Chiang, and K. C. Hwang, J. Mater. Chem. B 2, 1038 (2014).

90. Y. Li, G. Su, and Y. Tong, "Preparation of antitumor fluorescent diamond nanoparticleadriamycin composite for target drug delivery," CN 102716137 A 20121010 (2012). 
91. C. G. Reyes, L. J. C. Ricondo, G. A. A. Crespo, R. A. Lopez, and J. L. V. Navarro, "Nanoparticle conjugates for use as active principles in pharmaceuticals and cosmetics," ES 2376937 A1 20120321 (2012).

92. T. Petit, J.-C. Arnault, H. Girard, and R. Grall, Use of nanodiamands for generating therapeutic free radicals under irradiation, FR 2993180 A1 20140117, WO 2014009930, A1 20140116 (2014).
93. A. B. Vol'nova, D. N. Lenkov, S. K. Gordeev, and S. B. Korchagina, Method for targeted drug delivery into central nervous system of a body, RU 2479268 C2 20130420 (2013).

94. R. J. Yakovlev, System for delivering biologically active agents into an organism and method for producing said system, WO 2013015704 A1 2013013 (2013).

95. A. Razavi, Ionically functionalized nanodiamonds, US 20100239678 A1 20100923 (2010).

Received: 31 October 2013. Accepted: 24 January 2014. 